\title{
Les traductions de Claude Simon à la bibliothèque universitaire de Paris 8
}

Fanny Lambert

\section{(2) OpenEdition \\ 1 Journals}

Édition électronique

URL : http://journals.openedition.org/ccs/967

DOI : $10.4000 /$ ccs. 967

ISSN : 2558-782X

Éditeur :

Presses universitaires de Rennes, Association des lecteurs de Claude Simon

\section{Édition imprimée}

Date de publication : 30 avril 2015

Pagination : 199-202

ISBN : 9782753539990

ISSN : 1774-9425

\section{Référence électronique}

Fanny Lambert, « Les traductions de Claude Simon à la bibliothèque universitaire de Paris 8 », Cahiers Claude Simon [En ligne], 10 | 2015, mis en ligne le 22 septembre 2017, consulté le 19 avril 2019. URL : http://journals.openedition.org/ccs/967; DOI : 10.4000/ccs.967 


\title{
LES TRADUCTIONS DE CLAUDE SIMON À LA BIBLIOTHÈQUE UNIVERSITAIRE DE PARIS 8
}

\author{
Fanny LAMBERT \\ Responsable des fonds patrimoniaux, Université Paris 8
}

Depuis plusieurs années, la Bibliothèque de l'université Paris 8 Vincennes - Saint-Denis conserve un fonds Claude Simon, constitué d'imprimés déposés par l'Association des Lecteurs de Claude Simon. Si l'on y trouve des études autour de l'œuvre du Prix Nobel de littérature - monographies, thèses, périodiques - et une partie des éditions originales ou rééditions françaises, la plupart directement données par les époux Simon à l'Association des Lecteurs, l'originalité de ce fonds réside sans doute dans le grand nombre de traductions qui y sont conservées.

En effet, le fonds Claude Simon de l'université compte pas moins de 120 éditions différentes des traductions de Claude Simon dans 24 langues, dont une centaine ne se trouvant conservées dans aucune autre bibliothèque universitaire française, ce qui en fait un outil de travail unique en France. En comparaison, le fonds nordique de la Bibliothèque Sainte-Geneviève, avec sa dizaine de traductions en langues scandinaves, est la deuxième institution française à conserver le plus de traductions de Claude Simon. D'autres ouvrages ont été repérés dans certaines universités, mais ces dernières ne comptent pas plus d'un ou deux volumes ${ }^{1}$.

Claude Simon fut traduit très tôt - dès la fin des années 1950 dans certains pays - et réédité plusieurs fois. La bibliothèque conserve des traductions originales ou des rééditions de traductions publiées de 1959 à nos jours, le volume

1. Outre les éditions en danois, norvégien et suédois de la BSG, nous avons repéré deux traductions espagnoles à l'université de Perpignan, deux éditions espagnoles publiées au Venezuela à Rennes 2, dont la traduction de Gulliver; il existe également une édition étrangère dans les institutions suivantes: universités d'Angers, Bordeaux-Montaigne, Caen, Paris 3, Paris 4, ENS Lyon, BULAC, Strasbourg - BNU. 
le plus récent du fonds étant la publication en estonien de La Route des Flandres, parue à Tallinn en $2011^{2}$. Une partie non négligeable de ces ouvrages a été éditée en 1985 et 1986, soit lorsque Claude Simon obtint le Prix Nobel de littérature ${ }^{3}$.

Ainsi, il existe dans le fonds vingt éditions anglaises publiées entre 1959 et 2002, représentant 13 titres différents de Claude Simon. La plus ancienne est la traduction originale du Vent, établie par Richard Howard et publiée à New York en 1959. Six autres éditions conservées datent des années 1960, cinq ont été publiées dans la décennie 1970, quatre, dans les années 1980, et trois après 1990, la plus récente datant de 2002 (The trolley, New York, 2002), également par Richard Howard. En allemand, nous avons 26 éditions parues en Allemagne, en Autriche et en Suisse, représentant treize romans de Claude Simon. L'un des intérêts principaux de ce panel est que la bibliothèque possède, pour une partie de ces titres, deux traductions différentes. C'est le cas par exemple du Palace, dont nous possédons à la fois la traduction d'Elmar Tophoven, publiée pour la première fois à Münich en 1962, et celle d'Eva Moldenhauer, dans une édition de $2006^{4}$.

Les romans de Claude Simon firent également très tôt l'objet de traductions en langues nordiques. En Suède, celles de Carl Gustaf Bjurström, qui traduisit la presque totalité de son œuvre, ont été publiées à de nombreuses reprises de 1960 à nos jours. La bibliothèque possède neuf des traductions de Bjurström dans onze éditions différentes parues entre 1960 et 1994 . Le fonds comporte également sept éditions finnoises, six danoises et cinq norvégiennes. Enfin, la bibliothèque possède quelques éditions en langues d'Europe centrale et balkanique, dont par exemple les deux publications, en ex-Yougoslavie, des Géorgiques: la traduction slovène est publiée à Ljubljana en 1986; la traduction serbe est publiée à Belgrade en 1987.

2. C. Simon, Flandria tee, Tallinn, 2011

3. La remise du Prix Nobel à Claude Simon ne fut sans doute pas un hasard dans l'engouement que les éditeurs et les traducteurs marquèrent alors pour l'œuvre de Claude Simon.

4. La bibliothèque conserve également deux traductions différentes pour les titres suivants: Das Haar der Berenike (traductions de Silvia Ronelt et Eva Moldenhauer), Das Gras, (traductions d'Erika et Elmar Tophoven et Eva Moldenhauer), et Die Straße in Flandern (traductions d'Elmar Tophoven et Eva Moldenhauer). 


\begin{tabular}{|c|c|c|}
\hline Langue de traduction & Nombre d'éditions & Nombre de titres traduits \\
\hline Allemand & 26 & 13 \\
\hline Anglais & 20 & 14 \\
\hline Espagnol & 13 & 12 \\
\hline Suédois & 11 & 9 \\
\hline Finnois & 7 & 7 \\
\hline Italien & 6 & 6 \\
\hline Danois & 6 & 5 \\
\hline Norvégien & 5 & 4 \\
\hline Portugais & 5 & 4 \\
\hline Néerlandais & 4 & 3 \\
\hline Grec & 3 & 3 \\
\hline Roumain & 3 & 3 \\
\hline Catalan & 2 & 2 \\
\hline Chinois & 2 & 2 \\
\hline Polonais & 2 & 2 \\
\hline Russe & 2 & 2 \\
\hline Serbe & 2 & 2 \\
\hline Tchèque & 2 & 2 \\
\hline Arabe & 1 & 1 \\
\hline Bulgare & 1 & 1 \\
\hline Croate & 1 & 1 \\
\hline Estonien & 1 & 1 \\
\hline Slovaque & 1 & 1 \\
\hline Slovène & 1 & 1 \\
\hline
\end{tabular}

Les traductions de Claude Simon conservées à l'Université Paris 8

Toutefois, le fonds comporte certaines lacunes. Lacunes géographiques, tout d'abord, et particulièrement en ce qui concerne l'aire asiatique. Est-ce l'histoire de la bibliothèque, celle de l'Association des lecteurs ou peut-être les deux? Il est en tout cas à noter que les éditions asiatiques conservées dans le fonds n'étaient 
que peu nombreuses - deux traductions chinoises - avant un dépôt en 2014 de cinq éditions dans cette langue. De même, les traductions japonaises, pourtant précoces $^{5}$, sont totalement absentes, ainsi que les éditions parues en coréen.

L'aire méditerranéenne paraît également assez incomplète. Si le fonds comporte un nombre assez important de traductions espagnoles, on observe quelques manques importants en ce qui concerne l'italien, bien que nous conservions un échantillon représentatif. En revanche, aucune traduction de romans en arabe - le seul ouvrage en arabe que nous possédions étant une traduction du Discours de Stockholm - ni même en turc ou en albanais. En outre, le nombre de traductions serbes possédées par la bibliothèque - deux - est assez médiocre au regard du nombre de traductions publiées.

Autre lacune, l'absence totale - ou presque totale - des traductions de certains romans. S'il n'est pas surprenant de faire ce constat sur les premiers écrits de Claude Simon, beaucoup moins traduits que les suivants ${ }^{6}$, cela est plus étonnant concernant d'autres titres. L'exemple le plus remarquable en est Le Jardin des plantes, dont nous ne conservons aucune traduction; on peut aussi noter que $L e$ Tramway n'est conservé que dans trois langues étrangères: seules les traductions anglaise, danoise et finnoise sont présentes dans le fonds de la bibliothèque. Les traductions les plus récentes de ce roman en sont absentes, de même que la traduction allemande établie par Eva Moldenhauer et publiée en $2002^{7}$.

Ces lacunes ne doivent pas faire oublier la richesse de la collection de la bibliothèque. Ainsi, le fonds comporte 25 éditions des traductions de La Route des Flandres en 17 langues différentes - dont deux traductions en allemand ${ }^{8}$. Les Géorgiques sont présentes en 14 langues, auxquelles il faut ajouter la traduction d'extraits en polonais; 13 traductions du Palace y sont également conservées; 9 , de L'Acacia; 8, de l'Herbe; 7, d'Histoire et de L'Invitation.

La collaboration avec l'Association des Lecteurs de Claude Simon permettra sans doute d'enrichir encore cette collection, outil de recherche à la fois pour l'étude de l'œuvre de Claude Simon, du travail et des choix d'édition autour de ses romans à l'étranger, mais aussi de sa réception et de sa traduction dans le monde.

5. On peut ainsi noter que le roman Histoire, paru en français en 1967, fut traduit en japonais par Iwasaki Tsutomu et publié dès $1968 \mathrm{au}$ Japon. Édition absente du fonds de la bibliothèque, de même que les traductions d'Hiraoka Tokuyoshi, comme Le Tramway (Romen Densha), paru au Japon en 2003.

6. La bibliothèque conserve Das Seil, Frankfurt am Main, 1985 (traduction de La Corde raide) et La consagracion de la primavera: novela, Caracas, 1969 (traduction du Sacre du Printemps).

7. Claude Simon, Die Trambahn, Cologne, 2002

8. Die Straße in Flandern: Roman, Münich, 1961, pour la traduction d'Elmar Tophoven; Cologne, 2003, pour la traduction d'Eva Moldenhauer. 